\title{
Efficient Interleukin-21 Production by Optimization of Codon and Signal Peptide in Chinese Hamster Ovarian Cells
}

\author{
Hee Jun Cho ${ }^{1 \dagger}$, Byung Moo $\mathrm{Oh}^{1,3 \dagger}$, Jong-Tae Kim ${ }^{1}$, Jeewon Lim $^{1,3}$, Sang Yoon Park ${ }^{1}$, Yo Sep Hwang ${ }^{1,3}$, \\ Kyoung Eun Baek ${ }^{1}$, Bo-Yeon Kim ${ }^{2}$, Inpyo Choi ${ }^{1}$, and Hee Gu Lee ${ }^{1,3 *}$ \\ ${ }^{1}$ Immunotherapy Convergence Research Center, Korea Research Institute of Bioscience and Biotechnology (KRIBB), Daejeon 34141, Republic \\ of Korea \\ ${ }^{2}$ Anticancer Agent Research Center, Korea Research Institute of Bioscience and Biotechnology (KRIBB), Ochang, Cheongju 28116, Republic \\ of Korea \\ ${ }^{3}$ Department of Biomolecular Science, University of Science and Technology (UST), Daejeon 34113, Republic of Korea
}

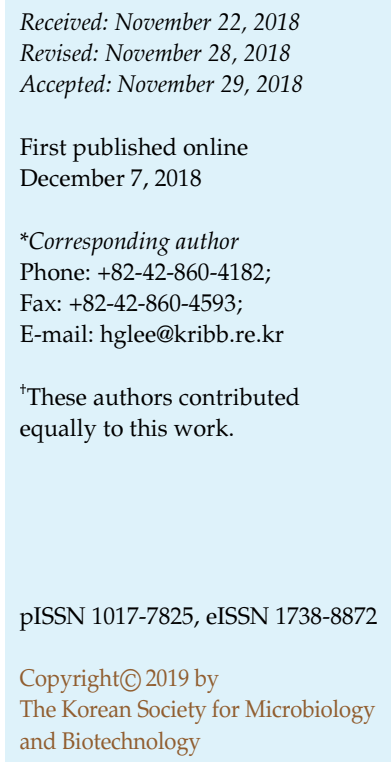

Interleukin-21 is a common $\gamma$-chain cytokine that controls the immune responses of B cells, $\mathrm{T}$ cells, and natural killer cells. Targeting IL-21 to strengthen the immune system is promising for the development of vaccines as well as anti-infection and anti-tumor therapies. However, the practical application of IL-21 is limited by the high production cost. In this study, we improved IL-21 production by codon optimization and selection of appropriate signal peptide in CHO-K1 cells. Codon-optimized or non-optimized human IL-21 was stably transfected into CHO-K1 cells. IL-21 expression was 10-fold higher for codon-optimized than non-optimized IL-21. We fused five different signal peptides to codon-optimized mature IL-21 and evaluated their effect on IL-21 production. The best result (a 3-fold increase) was obtained using a signal peptide derived from human azurocidin. Furthermore, codon-optimized IL-21 containing the azurocidin signal peptide promoted IFN- $\gamma$ secretion and STAT3 phosphorylation in NK-92 cells similar to codon-optimized IL-21 containing original signal peptide. Collectively, these results indicate that codon optimization and azurocidin signal peptides provide an efficient approach for the high-level production of IL-21 as a biopharmaceutical.

Keywords: IL-21, codon optimization, signal peptide, NK cells, CHO cells

\section{Introduction}

Interleukin-21 (IL-21) is a pleiotropic cytokine that regulates various immune responses [1]. IL-21 is predominantly produced in $\mathrm{CD}^{+} \mathrm{T}$ cells and natural killer $\mathrm{T}(\mathrm{NKT})$ cells. However, its receptor (IL-21R) is expressed in multiple cellular components of the innate and adaptive immune systems [2]. Therefore, it has potent immune regulatory properties in the context of infection, autoimmune diseases, and cancer [3-5]. IL-21 enhances the proliferation of CD4 ${ }^{+}$ $\mathrm{T}$ cells and regulates the development of IL-17-producing T-helper $\mathrm{T}$ cells, which are associated with autoimmune diseases and inflammatory conditions [6, 7]. IL-21 also enhances the proliferation and cytotoxicity of natural killer (NK) cells and promotes their interferon- $\gamma$ secretion $[8,9]$.
Although recombinant IL-21 (rhIL-21) therapy has been ongoing in preclinical studies and in Phase I and II clinical trials to treat patients with metastatic melanoma and renal cell cancer [10-12], the therapeutic applications of rhIL-21 are limited by the relatively high production cost.

There are several expression systems producing recombinant protein, such as bacteria, fungi, and mammalian cells. Mammalian cells are proper hosts for the production of recombinant proteins, because they are beneficial for posttranslational modifications (PTMs). Most marketed recombinant protein pharmaceuticals were produced by Chinese hamster ovary $(\mathrm{CHO})$ cells $[13,14]$. Gene optimization and the selection of an appropriate signal peptide can enhance the production of recombinant proteins in mammalian cells. A number of parameters, including codon usage, mRNA 
stability and the GC content, and RNA instability motifs and splicing sites, are considered for gene optimization to improve transcription, translation, and folding of recombinant protein $[15,16]$. The selection of proper signal peptides is also critical for establishing a manufacturing process for protein production to improve the correct processing and secretion of recombinant proteins through the transport of the translated proteins into the endoplasmic reticulum $[17,18]$. Recent studies have demonstrated the effectiveness of different signal peptides on the production of recombinant protein in $\mathrm{CHO}$ cells [19-22].

The aim of the current study was to improve the production of IL-21 in the CHO-K1 cell line. IL-21 codon optimization and the selection of a proper signal peptide improved productivity and maintained the biological properties of IL-21.

\section{Materials and Methods}

\section{Gene Optimization, Synthesis, and Vector Construction}

Codon optimization was performed using the Gene Optimization algorithm of Bioneer (Korea). Human IL-21 cDNA (BC066260) and all codon-optimized DNAs were synthesized and cloned into the pGH cloning vector (Bioneer). All genes encoding IL-21 were cloned into pcDNA3.1/zeo(+)-GS (24) at the AflII/BamHI site.

\section{Cell Culture and Transfections}

CHO-K1 cells were cultured in DMEM (-)Gln (11960-069; Thermo, USA) containing $10 \%$ dialyzed fetal bovine serum (dFBS, 26400-044; Thermo) and GS expression medium supplement (GSEM, G9785; Sigma-Aldrich, USA). Cells were transfected with a vector containing mock, non-optimized, and optimized human IL-21 genes using X-tremeGENE 9 DNA Transfection Reagent (06 365787 001; Roche, Basel, Switzerland) as described previously [23-25]. After transfection, $2 \times 10^{3}$ cells were seeded in 96-well plates and selected with DMEM (-)Gln containing 10\% dFBS, GSEM, $300 \mu \mathrm{g} / \mathrm{ml}$ zeocin (R250-01; Thermo), and $25 \mu \mathrm{M}$ GS System L-Methionine Sulfoximine (Sigma-Aldrich). IL-21 concentrations in the supernatants were measured when the cell pools reached confluence. To construct single cell clones, cell pools highexpressing non-optimized IL-21 or codon-optimized IL-21 were gradually expanded and subjected to limited dilution. IL-21 production in the respective clonal lines was measured by ELISA. The top three high-producing cell clones were used for further analyses.

\section{Signal Peptide Optimization}

Five different signal peptides were identified by literature search and fused with the codon-optimized mature IL-21 [16-19]. The origin and amino acid sequence of signal peptides are shown in Table 1.

\section{Quantification of IL-21 and IFN- $\gamma$ by ELISA}

IL-21 and IFN- $\gamma$ cytokines were evaluated in the culture supernatant using specific ELISA kits from Invitrogen [26-28]. NK92 cells were seeded in $5 \times 10^{5} / \mathrm{ml}$ with IL-2-free media for $24 \mathrm{~h}$. Following starvation, NK92 cells were stimulated with the commercial IL-21 expressed in bacteria (rhIL-21), original signal peptide fused-IL-21 (WT/IL-21), or Azurocidin signal peptidefused IL-21 (Az/IL-21) for $24 \mathrm{~h}$. IFN- $\gamma$ concentration was measured using specific ELISA kits (Invitrogen) according to the manufacturer's instructions.

\section{Western Blotting}

Western blot analysis was performed as described previously, with minor modifications $[29,30]$. In brief, cells were lysed in icecold lysis buffer (20 mmol/l Tris ( $\mathrm{pH} 8.0$ ), $137 \mathrm{mM} \mathrm{NaCl}, 10 \%$ glycerol, 1\% Nonidet P-40, $1 \mathrm{mM}$ phenylmethylsulfonyl fluoride, protease inhibitor mixture (Roche), $1 \mathrm{mM}$ sodium vanadate). Cell lysates were separated by SDS-PAGE and transferred to a polyvinylidene difluoride membrane (Amersham Biosciences, Amersham, UK). Subsequently, membranes were incubated in TBST supplemented with 5\% non-fat dry milk and probed with STAT3 (Cell Signalling Technology, USA) and phosphor-STAT3 (Cell Signalling Technology) antibodies at $4^{\circ} \mathrm{C}$ overnight. The bound antibodies were visualized with a suitable secondary antibody conjugated to horseradish peroxidase using enhanced chemiluminescence reagent (AB Frontier, Korea) [31, 32].

\section{Statistical Analysis}

Data were obtained from at least three independent experiments. All quantitative data are presented as means \pm standard deviations and were analyzed using Student's $t$-tests. $P<0.05$ was considered statistically significant.

Table 1. The origin and amino acid sequence of signal peptides.

\begin{tabular}{cllcl}
\hline Name & \multicolumn{1}{c}{ Signal peptide sequence } & \multicolumn{1}{c}{ Protein } & Accession No. & Organism \\
\hline HSA & MKWVTFISLLFLFSAYS & Human serum albumin & P02768 & Homo sapiens \\
RSA & MKWVTFLLLLFISGSAFS & Rat serum albumin & P02770 & Rattus norvegicus \\
AZ & MTRLTVLALLAGLLASSRA & Azurocidin preproprotein & NP_001691 & Homo sapiens \\
TPA & MDAMKRGLCCVLLLCGAVFVSP & Tissue-type plasminogen activator & P00750 & Homo sapiens \\
H7 & MEFGLSWVFLVALFRGVQC & Ig heavy chain signal peptide 7 & & \\
\hline
\end{tabular}



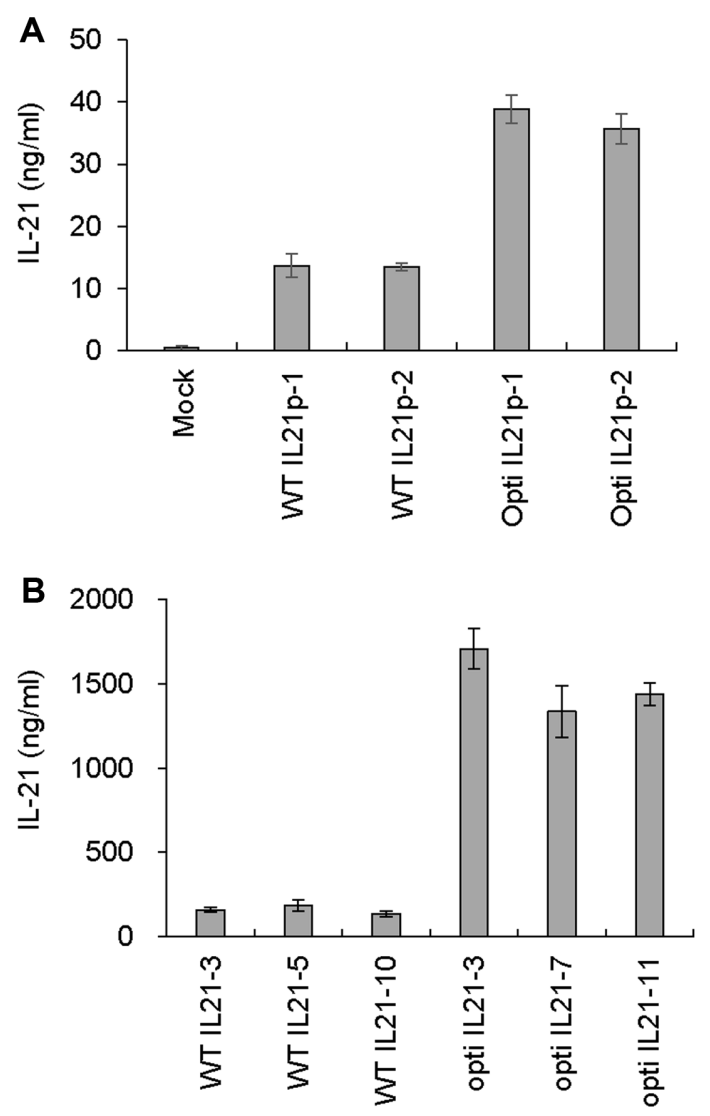

Fig. 1. Evaluation of IL-21 production by gene optimization. (A) CHO-K1 cells were transfected with the vector expressing WT or gene optimized IL-21. Two different CHO-K1-derived cell pools selected and defined were two WT IL21p (cell pools transfected with WT IL-21) and two opti IL-21p (cell pools transfected with opti IL-21). $1 \times 10^{5}$ cells were seeded in 6-well plates for 3 days. The concentration of IL-21 in the supernatant was measured by ELISA. (B) Three highproducing cell clones were constructed as described in Materials and Methods. $1 \times 10^{5}$ cells were seeded in 6-well plates for 3 days. The concentration of IL-21 in the supernatant was measured by ELISA.

\section{Results}

\section{Enhanced Production of IL-21 by Gene Optimization}

To investigate the effect of gene optimization on IL-21 expression in CHO-K1 cells, synthetic IL-21 genes were assembled based on codon usage in Chinese hamster (Cricetulus griseus) as described in Materials and Methods. Amino acid sequences for non-optimized IL-21 (WT IL-21) and optimized IL-21 (opti IL-21) were identical. The sequence of WT IL-21 and opti IL-21 had the identity of $82.4 \%$. The codon optimization index (COI) increased from 0.71 to 0.84 . The GC content was also adjusted from $40 \%$ to $51 \%$. To investigate the enhancement in productivity by gene optimization, $\mathrm{CHO}-\mathrm{K} 1$ cells were transfected with a vector containing WT or opti IL-21. Transfected cells were seeded in 96-well plates and selected. IL-21 concentrations in the culture supernatants were measured when the cell pools reached more than $90 \%$ confluence. Two cell pools with high production of WT or opti IL-21 were gradually expanded. The concentration of IL-21 in the supernatant was analyzed by ELISA when cells reached at least $90 \%$ confluence. Higher IL-21 concentrations were observed in two different cell pools transfected with optimized IL-21 (38.8 and $35.7 \mathrm{ng} / \mathrm{ml}$ ) than in two different cell pools transfected with non-optimized IL-21 (13.7 and $13.5 \mathrm{ng} / \mathrm{ml}$ ) (Fig. 1A). Next, cell pools were subjected to limiting dilution to establish single cell clones. The top three highproducing clones were selected. IL-21 secretion in respective clonal cell lines was analyzed by ELISA. The average yield of opti IL-21 (1,495 $\pm 192 \mathrm{ng} / \mathrm{ml})$ was 10-fold higher than that of WT IL-21 $(159 \pm 24 \mathrm{ng} / \mathrm{ml})$ (Fig. 1B). These results indicate that gene optimization enhances the production of IL-21 in CHO-K1 cells.

\section{Enhanced Secretion of IL-21 by the Azurocidin Signal Peptide}

Signal peptides play important roles in the secretion of proteins expressed in mammalian cells. To enhance the secretion of IL-21 in CHO-K1 cells, five promising signal peptides were selected by literature search [19-22]. Their origin and amino acid sequences are listed in Table 1. Five different signal peptides were fused to codon-optimized mature IL-21 (Fig. 2A). To evaluate the effects of signal peptides on IL-21 production, CHO-K1 cells were transfected with a vector expressing IL-21 fused to WT or to five different signals. Cell pools were selected as described in the Materials and Methods. IL-21 concentrations in the culture supernatants were analyzed by ELISA when the cells reached confluence. Although IL-21 production was greater using the rat serum albumin signal peptide (RSAP / mIL21) and tissue plasminogen activator signal peptide (tPASP/mIL21) than using the original signal peptide, the greatest productivity was observed for a cell pool expressing azurocidin signal peptide conjugated-IL-21 (AzSP/mIL21) (Fig. 2B). Although the azurocidin signal peptide improved IL-21 productivity in cell pools, the performance in stable cell lines can differ. Therefore, the AzSP/mIL21 cell pool was subjected to limiting dilution to generate stable single cell clones. IL-21 concentrations in the supernatants of twenty clones were measured by ELISA (data not shown). The top two clones with respect to IL-21 production (opti Az/IL21-5 and opti Az/IL21-12) were selected and subjected 
A

\begin{tabular}{rl|r|}
\cline { 2 - 3 } IL21SP/IL-21 & IL21SP & optimized mature IL-21 \\
\cline { 2 - 3 } HSASP/mIL21 & HSASP & optimized mature IL-21 \\
\cline { 2 - 3 } RSASP/mIL21 & RSASP & optimized mature IL-21 \\
\cline { 2 - 3 } AzSP/mIL21 & AzSP & optimized mature IL-21 \\
\cline { 2 - 3 } tPASP/mIL21 & & optimized mature IL-21 \\
\cline { 2 - 3 } H7SP/mIL21 & tPASP & optimized mature IL-21
\end{tabular}
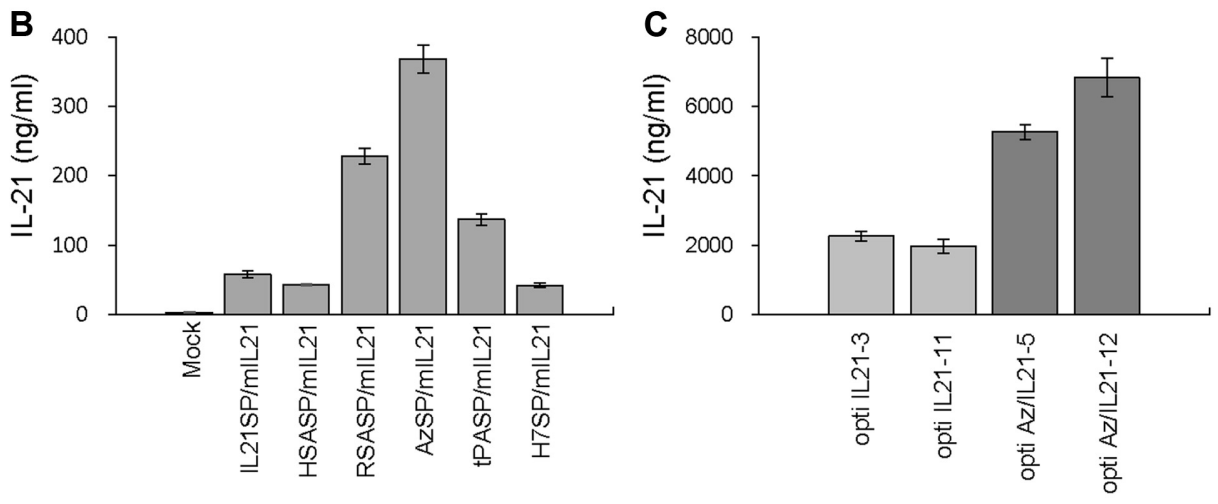

Fig. 2. Enhanced production of IL-21 by the azurocidin signal peptide.

(A) WT or five different signal peptides were fused to codon-optimized mature IL-21. (B) CHO-K1 cells were transfected with the vector expressing IL-21 fused to WT or five indicated signal peptides. Cell pools were selected as described in Materials and Methods. $1 \times 10^{5}$ cells were seeded in 6-well plates for 3 days. The concentration of IL-21 in the supernatant was measured by ELISA. (C) Two high-producing cell clones were constructed as described in Materials and Methods. $1 \times 10^{5}$ cells were seeded in 6-well plates for 3 days. The concentration of IL-21 in the supernatant was measured by ELISA.

to ELISA with two clones producing the optimized original signal peptide IL-21 (opti IL21-3 and opti IL21-11). Based on ELISA, opti Az/IL21-5 and opti Az/IL21-12 cell clones resulted in a more than 2.5-fold increase in the IL-21 concentration compared to the concentrations for opti IL213 and opti IL21-11 cell clones (Fig. 2C). These data suggest that the azurocidin signal peptide enhances the secretion of IL-21 in CHO-K1 cells.

\section{Biological Activities of Codon- and Signal Peptide- Optimized IL-21}

IL-21 induces the phosphorylation of STAT3 and promotes the secretion of interferon- $\gamma$ (IFN- $\gamma$ ) in NK cells [2, 9]. To verify the biological activity of optimized IL-21, NK-92 cells were stimulated with various concentrations of commercial IL-21 expressed in bacteria (rhIL-21), original signal peptide IL-21 (WT/IL-21), or azurocidin signal peptide IL-21 (Az/IL-21). After $24 \mathrm{~h}$, secreted IFN- $\gamma$ in the culture supernatant was measured by ELISA. WT/IL-21 and Az/IL-21 produced by CHO-K1 cells showed similar induction of the secretion of IFN- $\gamma$ in NK-92 cells (Fig. 3A). However, commercial rhIL-21 produced by bacteria resulted in lower levels of IFN- $\gamma$ production than those for
WT/IL-21 and Az/IL-21 in mammalian cells (Fig. 3A). Furthermore, western blot analysis showed that Az/IL-21 induced strong STAT3 phosphorylation, even at low concentrations, whereas rhIL-21 resulted in a slight increase in the phosphorylation of STAT3 (Fig. 3B). These data suggest that Az/IL-21 has similar biological effects on IFN- $\gamma$ production and STAT3 phosphorylation to those of WT/IL-21 and better activity than those of commercial rhIL-21.

\section{Discussion}

Most bio-therapeutics are produced in established mammalian cell lines, like $\mathrm{CHO}$ cells, which ensure correct glycosylation for the modulation of pharmaceutical efficacy in vivo. However, high production costs by low productivity and time requirements by slow proliferation limit the development and use of these systems [33]. Recently, many studies have aimed to increase the production of recombinant proteins in $\mathrm{CHO}$ systems by manipulating various factors [34, 35]. Expression vector engineering technologies, including codon optimization of the gene of interest and selection of appropriate signal 


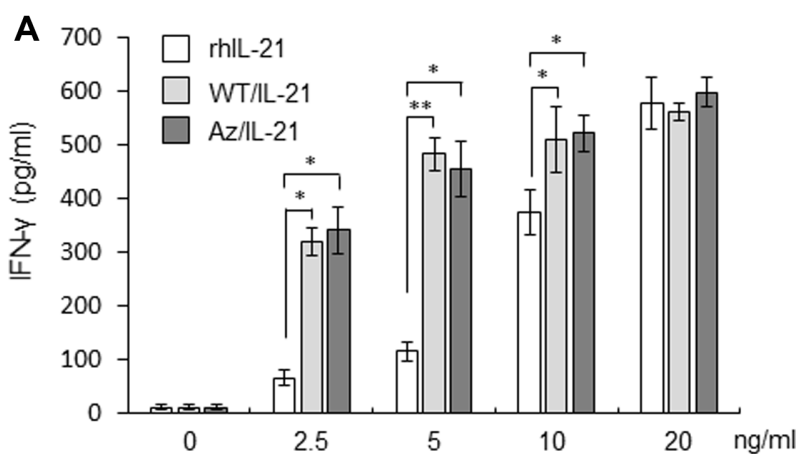

B

$\begin{array}{rlllll}\text { rhlL-21 (ng) } & - & 1 & - & 2.5 & - \\ \text { Az/lL-21 (ng) } & - & - & 1 & - & 2.5 \\ \text { p-STAT3 } & & & \\ & & & & & \end{array}$

Fig. 3. IFN- $\gamma$ production and STAT3 phosphorylation by recombinant IL-21 proteins in human NK-92 cells.

(A) NK-92 cells were serum-starved for $18 \mathrm{~h}$ and then treated with indicated concentrations of rhIL-21, WT/IL-21, or Az/IL-21 for $24 \mathrm{~h}$. The amount of secreted IFN- $\gamma$ in the culture supernatant was measured by ELISA. Data represent the mean \pm S.D. of three individual experiments. ${ }^{*}, p<0.05,{ }^{* *}, p<0.01$. (B) Serum-starved NK-92 cells were treated with rhIL-21 or Az/IL-21 for $30 \mathrm{~min}$. Cell lysates were immunoblotted with pSTAT3 or STAT3 antibodies.

peptides, are the most convenient methods to enhance productivity $[15,19]$.

Previous studies have shown that optimization of various factors, including codon usage bias, codon context, GC content, premature poly A, cryptic spice sites, and CpG dinucleotide content [36]. Our results showed that IL-21 productivity was improved 10 -fold by gene optimization (Fig. 2B). Many studies have shown that gene optimization is a powerful method to enhance productivity by improving transcription, translation, and protein folding in nonmammalian or mammalian expression systems [37]. The GC content directly regulates the stability of mRNA and indirectly affects the mRNA level [15]. In this study, gene optimization of $I L-21$ resulted in an increase in the COI ( 0.71 to 0.84 ), which is a measure of codon usage bias, as well as in the GC content ( $40 \%$ to $51 \%$ ). Therefore, increased production resulting from gene optimization may be attributed the enhancement of both translation and mRNA level.

Signal peptides regulate efficient transport to the ER, appropriate folding, PTM, and the secretion of translated proteins to the culture supernatant. Several studies suggest that the production of recombinant protein can be improved by the use of alternative signal peptides [19-22]. In this study, we evaluated the efficiency of five signal peptides on the secretion of IL-21. Three signal peptides (derived from rat serum albumin, azurocidin, and tissue plasminogen activator signal peptides) resulted in higher productivity than that of the original signal peptide, whereas two signal peptides (derived from human serum albumin and H7 signal peptide) were similar to the control peptide with respect to productivity. Most notably, the azurocidin signal peptide showed the highest IL-21 production activity (Fig. 2B). Consistent with our results, a previous study used the azurocidin signal peptide to potently express various proteins in mammalian cells [21]. However, another study demonstrated that the human albumin signal peptide shows the highest secretion activity for the production of recombinant protein among various signal peptides [22]. This apparent discrepancy may be attributed to differences in experimental design or systems. Results may vary depending on the cell line or organism. Furthermore, the secretion efficiency can be affected by the amino acid sequence downstream of a signal peptide [38, 39].

The biological activities of recombinant IL-21 produced by CHO-K1 cells were assessed by measuring IFN- $\gamma$ secretion and STAT3 phosphorylation in NK-92 cells (Fig. 3). Codon-optimized Az/IL-21 (IL-21 secreted by the azurocidin-derived signal peptide) had similar activity to that of codon-optimized WT/IL-21 (IL-21 secreted by the original signal peptide). Thus, although the azurocidinderived signal peptide increases IL-21 production (Fig. 2), it does not affect the biological activity of IL-21 toward NK cells. However, the biological activity of both Az/IL-21 and WT/IL-21 in CHO-K1 increased compared to that of commercial recombinant IL-21 produced in bacteria (Fig. 3). $\mathrm{CHO}$ cells regulate PTMs to improve biological activity and reduce the immunological response to the therapeutic recombinant protein. Proper folding of secreted proteins by disulphide bonds, which can be obtained in mammalian cells, is important for their activity $[40,41]$. Therefore, these enhanced activities can be explained by PTMs, such as glycosylation, sialyation, and fucosylation, or proper protein folding.

In conclusion, we demonstrated that codon optimization and Azurocidin signal peptide can improve the production efficiency of human IL-21, which has biological activity, in CHO-K1 cells. Our results provide a potential strategy for the production of recombinant IL-21 as a biopharmaceutical. 


\section{Acknowledgments}

This research was supported by the National Research Council of Science and Technology (NST) grant by the Korea government (CRC-15-02-KRIBB) and the National Research Foundation of South Korea (NRF) (No. 2018R1A2B6005472).

\section{Conflict of Interest}

HJ Cho, BY Kim, I Choi, and HG Lee have pending patent applications related to this study. Other authors have no conflict of interest to declare.

\section{References}

1. Rochman Y, Spolski R, Leonard WJ. 2009. New insights into the regulation of $\mathrm{T}$ cells by gamma(c) family cytokines. Nat. Rev. Immunol. 9: 480-490.

2. Spolski R and Leonard WJ. 2014. Interleukin-21: a doubleedged sword with therapeutic potential. Nat. Rev. Drug Discov. 13: 379-95.

3. Asao H, Okuyama C, Kumaki S, Ishii N, Tsuchiya S, Foster D, et al. 2001. Cutting edge: the common $\gamma$-chain is an indispensable subunit of the IL-21 receptor complex. J. Immunol. 167: 1-5.

4. Kang S, Myoung J. 2017. Host innate immunity against hepatitis E virus and viral evasion mechanisms. J. Microbiol. Biotechnol. 27: 1727-1735.

5. Kang S, Myoung J. 2017. Primary lymphocyte infection models for KSHV and its putative tumorigenesis mechanisms in B cell lymphomas. J. Microbiol. 55: 319-329.

6. Spolski R, Leonard WJ. 2008. Interleukin-21: basic biology and implications for cancer and autoimmunity. Annu. Rev. Immunol. 26: 57-79.

7. Spolski R, Leonard WJ. 2008. The Yin and Yang of interleukin-21 in allergy, autoimmunity and cancer. Curr. Opin. Immunol. 20: 295-301.

8. Parrish-Novak J, Dillon SR, Nelson A, Hammond A, Sprecher C, Gross JA, et al. 2000. Interleukin 21 and its receptor are involved in NK cell expansion and regulation of lymphocyte function. Nature 408: 57-63.

9. Park YK, Shin DJ, Cho D, Kim SK, Lee JJ, Shin MG et al. 2012. Interleukin-21 increases direct cytotoxicity and IFN- $\gamma$ production of ex vivo expanded NK cells towards breast cancer cells. Anticancer Res. 32: 839-846.

10. Davis ID, Skrumsager BK, Cebon J, et al. 2007. An open-label, two-arm, phase I trial of recombinant human interleukin-21 in patients with metastatic melanoma. Clin. Cancer Res. 13: 3630-3636.

11. Thompson JA, Curti BD, Redman BG, et al. 2008. Phase I study of recombinant interleukin-21 in patient with metastatic melanoma and renal cell carcinoma. J. Clin. Oncol. 26: 20342039.

12. Petrella TM, Tozer R, Belanger K, et al. 2012. Interleukin-21 has activity in patients with metastatic melanoma: a phase II study. J. Clin. Oncol. 30: 3396-3401.

13. Zhu J. 2012. Mammalian cell protein expression for biopharmaceutical production. Biotechnol. Adv. 30: 1158-1170.

14. Walsh G. 2014. Biopharmaceutical benchmarks. Nat. Biotechnol. 32: 992-1000.

15. Khan KH. 2013. Gene expression in Mammalian cells and its applications. Adv. Pharm. Bull. 3: 257-263.

16. Hung F, Deng L, Ravnikar P, Condon R, Li B, Do L, et al. 2010. mRNA stability and antibody production in $\mathrm{CHO}$ cells: improvement through gene optimization. Biotechnol. J. 5: 393-401.

17. You M, Yang Y, Zhong C, Chen F, Wang X, Jia T, et al. 2018. Efficient $\mathrm{mAb}$ production in $\mathrm{CHO}$ cells with optimized signal peptide, codon, and UTR. Appl. Microbiol. Biotechnol. doi: 10.1007/s00253-018-8986-5. [Epub ahead of print]

18. Jeiranikhameneh M, Moshiri F, Keyhan Falasafi S, Zomorodipour A. 2017. Designing signal peptides for efficient periplasmic expression of human growth hormone in Escherichia coli. J. Microbiol. Biotechnol. 27: 1999-2009.

19. Attallah C, Etcheverrigaray M, Kratje R, Oggero M. 2017. A highly efficient modified human serum albumin signal peptide to secrete proteins in cells derived from different mammalian species. Protein Expr. Purif. 132: 27-33.

20. Haryadi R, Ho S, Kok YJ, Pu HX, Zheng L, Pereira NA, et al. 2015. Optimization of heavy chain and light chain signal peptides for high level expression of therapeutic antibodies in CHO cells. PLoS One 10: e0116878.

21. Kober L, Zehe C, Bode J. 2013. Optimized signal peptides for the development of high expressing $\mathrm{CHO}$ cell lines. Biotechnol. Bioeng. 110: 1164-1173.

22. Knappskog S, Ravneberg H, Gjerdrum C, Trösse C, Stern B, Pryme IF. 2007. The level of synthesis and secretion of Gaussia princeps luciferase in transfected $\mathrm{CHO}$ cells is heavily dependent on the choice of signal peptide. J. Biotechnol. 128: 705-715.

23. Cho HJ, Kim JT, Lee SJ, Hwang YS, Park SY, Kim BY, et al. 2018. Protein phosphatase 1B dephosphorylates Rho guanine nucleotide dissociation inhibitor 1 and suppresses cancer cell migration and invasion. Cancer Lett. 417: 141-151.

24. Shin JS, Ku KB, Jang Y, Yoon YS, Shin D, Kwon OS, et al. 2017. Comparison of anti-influenza virus activity and pharmacokinetics of oseltamivir free base and oseltamivir phosphate. J. Microbiol. 55: 979-983.

25. Kim JH, Lee CH, Lee SW. 2016. Hepatitis C virus infection stimulates transforming growth factor-beta1 expression through up-regulating miR-192. J. Microbiol. 54: 520-526.

26. Hong S, Yu JW. 2018. Prolonged exposure to lipopolysaccharide induces NLRP3-independent maturation and secretion of 
interleukin (IL)-1 $\beta$ in macrophages. J. Microbiol. Biotechnol. 28: 115-121.

27. Bhardwaj M, Cho HJ, Paul S, Jakhar R, Khan I, Lee SJ et al. 2017. Vitexin induces apoptosis by suppressing autophagy in multi-drug resistant colorectal cancer cells. Oncotarget. 9: 3278-3291.

28. Choi DW, Jung SY, Kang J, Nam YD, Lim SI, Kim KT et al. 2018. Immune-enhancing effect of nanometric Lactobacillus plantarum $\mathrm{nF1}(\mathrm{nLp}-\mathrm{nF} 1)$ in a mouse model of cyclophosphamideinduced immunosuppression. J. Microbiol. Biotechnol. 28: 218226.

29. Cho HJ, Hwang YS, Yoon J, Lee M, Lee HG, Daar IO. 2018. EphrinB1 promotes cancer cell migration and invasion through the interaction with RhoGDI1. Oncogene 37: 861-872.

30. Yoon J, Hwang YS, Lee M, Sun J, Cho HJ, Knapik L, et al. 2018. TBC1d24-ephrinB2 interaction regulates contact inhibition of locomotion in neural crest cell migration. Nat. Commun. 9: 3491 .

31. Kim E, Myoung J. 2018. Hepatitis E virus papain-like cysteine protease inhibits type I interferon induction by down-regulating melanoma differentiation-associated gene 5 . J. Microbiol. Biotechnol. 28: 1908-1915.

32. Kang S, Choi C, Choi I, Han KN, Rho SW, Choi J, et al. 2018. Hepatitis E virus methyltransferase inhibits type I interferon induction by targeting RIG-I. J. Microbiol. Biotechnol. 28: 1554-1562.

33. Lai T, Yang Y, Ng SK. 2013. Advances in Mammalian cell line development technologies for recombinant protein production. Pharmaceuticals 6: 579-603.

34. Schaub J, Clemens C, Schorn P, Hildebrandt T, Rust W,
Mennerich D, et al. 2010. CHO gene expression profiling in biopharmaceutical process analysis and design. Biotechnol. Bioeng. 105: 431-438.

35. Reinhart D, Damjanovic L, Kaisermayer C, Kunert R. 2015. Benchmarking of commercially available $\mathrm{CHO}$ cell culture media for antibody production. Appl. Microbiol. Biotechnol. 99: 4645-4657.

36. Han JH, Choi YS, Kim WJ, Jeon YH, Lee SK, Lee BJ, et al. 2010. Codon optimization enhances protein expression of human peptide deformylase in E. coli. Protein Expr. Purif. 70: 224-230.

37. Fath S, Bauer AP, Liss M, Spriestersbach A, Maertens B, Hahn P, et al. 2011. Multiparameter RNA and codon optimization: a standardized tool to assess and enhance autologous mammalian gene expression. PLoS One 6: e17596.

38. Andrews DW, Perara E, Lesser C, Lingappa VR. 1988. Sequences beyond the cleavage site influence signal peptide function. J. Biol. Chem. 263: 15791-15798.

39. Wiren KM, Potts JT Jr, Kronenberg HM. 1988. Importance of the propeptide sequence of human preproparathyroid hormone for signal sequence function. J. Biol. Chem. 263: 19771-19777.

40. Butler M, Spearman M. 2014. The choice of mammalian cell host and possibilities for glycosylation engineering. Curr. Opin. Biotechnol. 30: 107-112.

41. Zhang P, Chan KF, Haryadi R, Bardor M, Song Z. 2013. $\mathrm{CHO}$ glycosylation mutants as potential host cells to produce therapeutic proteins with enhanced efficacy. Adv. Biochem. Eng. Biotechnol. 131: 63-87. 